\title{
Disease progression continues in patients with advanced Parkinson's disease and effective subthalamic nucleus stimulation
}

\author{
R Hilker*, A T Portman*, J Voges, M J Staal, L Burghaus, T van Laar, A Koulousakis, R P Maguire, \\ J Pruim, B M de Jong, K Herholz, V Sturm, W-D Heiss, K L Leenders
}

See Editorial Commentary, p 1186

J Neurol Neurosurg Psychiatry 2005;76:1217-1221. doi: 10.1136/jnnp.2004.057893

*The first two authors

contributed equally to this

work.

See end of article for authors' affiliations

......................

Correspondence to:

Dr R Hilker, Department of

Neurology, University

Hospital, Joseph-

Stelzmann-Strasse 9,

50924 Cologne,

Germany; hilker@pet.

mpin-koeln.mpg.de

Received 3November 2004

Revised version received

14 December 2004

Accepted

16 December 2004

\begin{abstract}
Objectives: Glutamate mediated excitotoxicity of the hyperactive subthalamic nucleus (STN) has been reported to contribute to nigral degeneration in Parkinson's disease (PD). Deep brain stimulation of the STN (STN DBS), in its role as a highly effective treatment of severe PD motor complications, has been thought to inhibit STN hyperactivity and therefore decrease progression of PD.

Methods: In a prospective two centre study, disease progression was determined by means of serial ${ }^{18} \mathrm{~F}$ fluorodopa (F-dopa) positron emission tomography (PET) in 30 patients with successful STN DBS over the first 16 (SD 6) months after surgery.

Results: Depending on the method of PET data analysis used in the two centres, annual progression rates relative to baseline were $9.5-12.4 \%$ in the caudate and $10.7-12.9 \%$ in the putamen.

Conclusions: This functional imaging study is the first to demonstrate a continuous decline of dopaminergic function in patients with advanced PD under clinically effective bilateral STN stimulation. The rates of progression in patients with STN DBS were within the range of previously reported data from longitudinal imaging studies in PD. Therefore this study could not confirm the neuroprotective properties of DBS in the STN target.
\end{abstract}

previously to quantify presynaptic dopaminergic function in human subjects. ${ }^{14-16}$ Here we report the results of the first prospective study on the progression of PD after successful STN DBS measured with ${ }^{18}$ F-fluorodopa (F-dopa) PET.

\section{PATIENTS AND METHODS}

Clinical evaluation of patients and surgical procedure A total of 30 patients with advanced PD (19 men, 11 women; mean age (SD) 59.8 (7.2) years; mean disease duration 12.6 (4.2) years; mean Hoehn and Yahr stage ${ }^{17}$ off-drug 3.6 (0.5); 26 akinetic rigid PD, 4 tremor dominant PD) were consecutively recruited during the years 1999-2003 after obtaining written informed consent according to the Declaration of Helsinki. The patients were prospectively followed up at two centres - the neurological departments of Cologne University, Germany, and Groningen University, the Netherlands. The local ethics committees of both medical faculties approved the study. We diagnosed PD according to the UK Parkinson's Disease Society Brain Bank criteria, ${ }^{18}$ and indication for STN DBS according to the CAPSIT-PD protocol, ${ }^{19}$ - that is, presence of parkinsonian symptoms refractory to medication, such as severe levodopa associated on-off fluctuations, peak dose dyskinesias, and severe resting hand tremor. All study patients had a clear but short levodopa response and did not show any atypical clinical signs.

We implanted bilateral STN electrodes (Medtronic model 3389, Medtronic, Minneapolis, MN) and impulse generators (Itrel II or Kinetra, Medtronic $\mathrm{GmbH}$ ) in each patient. Both

Abbreviations: DBS, direct brain stimulation; $\mathrm{K}_{\text {occ, }}$ influx constant; LEDD, levodopa equivalent daily dose; PET, positron emission tomography; ROI, region of interest; SOR, striatal to occipital ratio; STN, subthalamic nucleus; UPDRS, Unified Parkinson's Disease Rating Scale positron emission tomography (PET) or single photon emission computed tomography (SPECT) have been used 
centres followed similar surgical procedures (departments of stereotaxy and functional neurosurgery of Cologne and Groningen universities) described in detail previously. ${ }^{20}{ }^{21}$ The targets were verified intraoperatively by microstimulation (Groningen) and macrostimulation (Groningen, Cologne) and regular neurological monitoring of clinical DBS effects (Groningen, Cologne). We used the Unified Parkinson's Disease Rating Scale (UPDRS) ${ }^{22}$ for clinically measuring disease severity before and after surgery as follows.

- Before surgery:

- in the drug-off condition 12 hours after cessation of antiparkinson medication ("practically defined off")

- one to two hours after the oral intake of 150-300 mg soluble levodopa ("best on").

- After surgery:

- under continuous STN stimulation (DBS-on condition) six months after electrode implantation

- at the time of the follow up PET scan (DBS-on condition).

The levodopa equivalent daily dose (LEDD) was calculated on the basis of the following formula ${ }^{4}: 1 \mathrm{mg}$ of pergolide = $1 \mathrm{mg}$ of lisuride $=1 \mathrm{mg}$ of pramipexole $=2 \mathrm{mg}$ of cabergoline $=5 \mathrm{mg}$ of ropinirole $=10 \mathrm{mg}$ of bromocriptine $=$ $5 \mathrm{mg}$ of apomorphine $=20 \mathrm{mg}$ of dihydroergocriptine $=$ $100 \mathrm{mg}$ levodopa. To assure correct electrode placement in the study sample, only those patients with a favourable stimulation effect-that is, with more than 30\% improvement of the UPDRS III score in the DBS-on versus off condition six months after surgery-were included in the PET follow up protocol.

\section{PET data acquisition}

Each patient had one baseline scan 4 (SD 6) weeks (range 114) prior to STN electrode implantation and one follow up scan at least one year after the beginning of STN DBS with the same PET camera, following the same study protocol. The mean (SD) follow up interval was 16 (6) months (range 1236). At both study centres, two 24 detector ring scanners (ECAT EXACT HR and ECAT EXACT, Siemens-CTI, Knoxville, TN) were used. ${ }^{23}{ }^{24}$ The patients were positioned supine in a relaxed state with their eyes closed and ears unplugged. To avoid drug interference, the antiparkinson medication was stopped for a minimum of 12 hours prior to the PET scanning procedure (drug-off condition). The follow up scan was performed in the DBS-on and drug-off condition (see table 1 for the DBS parameters). After pretreatment with $100 \mathrm{mg}$ carbidopa to block peripheral dopa-decarboxylase activity, 150-370 MBq of F-dopa was injected intravenously. Following this, at the Cologne centre, all patients $(n=20)$ were scanned with a dynamic series of nine 10 minutes frames over 90 minutes in a three dimensional mode, whereas at the Groningen centre, the first three patients had a static scan protocol which consisted of a single scan from 90-120 minutes after the injection. The remaining seven patients had a dynamic scan protocol with 21 time frames of increasing duration over a period of 120 minutes. Images were corrected for scatter and attenuation. We realigned individual frames from all dynamic scans to get a summed image from 0 to 90 minutes and from 0 to 120 minutes to reduce possible motion artefacts.

\section{PET data analysis}

We analysed the data on SUN Sparc 2 workstations (Sun Microsystems, Silicon Valley, CA). For each patient, both PET scans were analysed at the same time following a standardised protocol including one rater blinded for the presence of baseline or follow up PET scans. Four regions of interest (ROIs) were placed on the summed axial F-dopa images, namely the right and left head of the caudate nucleus and the right and left putamen. Reference regions were defined over the occipital cortex. In Cologne, both PET scans were coaligned exactly with standard software (MPI-tool). ${ }^{25}$ Then the ROI set was placed by inspection of the coaligned summed images and slightly adapted to the individual basal ganglia anatomy. In Groningen, the PET volume data were first linearly normalised to the Talairach coordinate space using the regional cerebral blood flow ( $\mathrm{rCBF}$ ) template of the SPM99 software package (SPM99, Wellcome Department of Cognitive Neurology, London, UK). Then ROI analysis was based on a standard template fixed in Talairach stereotactic space.

We used two previously validated methods of striatal Fdopa uptake determination, the striatal to occipital ratio (SOR) method (patients from both centres) and the influx constant $\left(\mathrm{K}_{\mathrm{occ}}\right)$ calculated by the multiple time graphical analysis (MTGA) method (patients from Cologne only). ${ }^{26}$ For SOR calculation, the mean ROI activity concentration was computed in either static scans or the averaged last two frames of dynamic scans. The SOR index was expressed as:

$$
\mathrm{SOR}=\mathrm{C}_{\mathrm{ROI}}-\mathrm{C}_{\mathrm{REF}} / \mathrm{C}_{\mathrm{REF}}
$$

where $\mathrm{C}_{\mathrm{ROI}}=$ average $\mathrm{ROI}$ activity concentration and $\mathrm{C}_{\mathrm{REF}}=$ average occipital activity concentration in the occipital reference region. For MTGA analysis, time activity curves of dynamic PET series were plotted with the occipital reference region activity serving as input function resulting in the striatal F-dopa influx constant $\mathrm{K}_{\mathrm{occ}}\left(\mathrm{min}^{-1}\right)$ which represents the rate of striatal F-dopa uptake and storage. ${ }^{27}$

\section{Calculation of disease progression and statistical analysis}

Right and left striatal F-dopa $\mathrm{K}_{\mathrm{occ}}$ and SOR values were averaged for each patient. Within each ROI, the annual decline of F-dopa $\mathrm{K}_{\mathrm{occ}}$ and SOR over the entire study period was calculated as the difference of baseline minus follow up PET data divided by the duration of the individual PET follow up period. For each individual and each ROI, annual progression rates were then expressed as the percentage of baseline $\mathrm{K}_{\mathrm{occ}}$ and SOR values. Paired Student's $t$ test was used to evaluate intraindividual differences between baseline and follow up $\mathrm{K}_{\mathrm{occ}}$ and SOR values and between UPDRS scores. Statistical significance was accepted at the level of $\mathrm{p}<0.05$ ( SPSS for Windows 10.0, SPSS UK Ltd, Surrey, England).

\section{RESULTS}

At the time of the follow up PET scan, we found a significant clinical improvement caused by STN DBS, that is, a 52\% decrease of the drug-off/DBS-on UPDRS motor score compared with the corresponding drug-off baseline value $(\mathrm{p}<0.001$; see table 1$)$. The LEDD was significantly reduced by $28 \%$ from before surgery to the follow up PET investigation $(\mathrm{p}<0.001$; see table 1$)$. A dopamine agonist was also continued in most patients after surgery (agonist medication in $27 / 30$ patients $(90 \%)$ at baseline and in 24/30 patients $(80 \%)$ at time of follow up PET). During the follow up period, the drug-off/DBS-on UPDRS motor score at the PET follow up was nearly unchanged from that at the six month follow up $(\mathrm{p}=0.4$, paired $t$ test; see table 1$)$. In contrast, a significant improvement occurred in the corresponding drug-on value at six months versus baseline $(p<0.05$; see table 1$)$. However, this was lost at the PET follow up ( $v$ six month scores $\mathrm{p}<0.01$; $v$ baseline $\mathrm{p}=0.7$; see table 1 ). Data on clinical outcome and STN DBS parameters are also summarised in table 1.

Both PET investigations showed a similar F-dopa uptake reduction pattern with the putamen more severely affected than 
Table 1 Clinical outcome and STN DBS parameters $(n=30$; values are mean (SD))

\begin{tabular}{|c|c|c|c|}
\hline & \multirow{2}{*}{$\frac{\text { Before surgery }}{\text { Baseline F-dopa PET }}$} & \multicolumn{2}{|l|}{ After surgery } \\
\hline & & At six month follow up & $\begin{array}{l}\text { At follow up F-dopa PET } \\
\text { (12-36 months) }\end{array}$ \\
\hline \multicolumn{4}{|l|}{ UPDRS III score } \\
\hline Drug-off condition & $42.9(11.4)^{*}$ & $19.4(9.1) \ddagger^{* *}$ & $20.4(8.4) \ddagger^{\star *}$ \\
\hline Drug-on condition & $16.5(7.6)+\oplus$ & $13.8(6.9) \S+\dagger$ & $16.0(7.4) \S \neq \ddagger$ \\
\hline LEDD (mg) & $935(384)$ & $611(288)^{* *}$ & $670(324)^{* *}$ \\
\hline \multicolumn{4}{|l|}{ DBS parameters } \\
\hline Amplitude (V) & - & $3.2(0.9)$ & $3.4(0.7)$ \\
\hline Pulse width ( $\mu$ s) & - & $72.5(14.3)$ & $72.5(14.2)$ \\
\hline \multirow{3}{*}{$\begin{array}{l}\text { Frequency }(\mathrm{Hz}) \\
\text { Mode }\end{array}$} & - & $133.9(10.2)$ & $137.1(14.5)$ \\
\hline & & Monopolar ( $n=58$ & Monopolar ( $n=58$ electrodes) \\
\hline & - & $\begin{array}{l}\text { electrodes) } \\
\text { Bipolar ( } n=2 \text { electrodes) }\end{array}$ & Bipolar ( $n=2$ electrodes) \\
\hline
\end{tabular}

*Obtained at least 12 hours after cessation of antiparkinsonian medication ("practically defined off"). †Obtained after oral intake of $200 \mathrm{mg}$ soluble levodopa ("best on").

fObtained at least 12 hours after cessation of antiparkinsonian medication under continuous STN stimulation (DBS-on).

§Obtained after oral intake of $200 \mathrm{mg}$ soluble levodopa under continuous STN stimulation (DBS-on).

- Difference between baseline drug-off and drug-on conditions: $p<0.001$ (paired $t$ test).

**Difference between drug-off baseline and follow up scores: $p<0.001$ (paired $t$ test).

$t+$ Difference between drug-on baseline and follow up scores: $p<0.05$ (paired $t$ test).

$\ddagger \mp D$ ifference between drug-on follow up scores: $p<0.01$ (paired $t$ test).

DBS, direct brain stimulation; LEDD, levodopa equivalent daily dose; PET, positron emission tomography; STN,

subthalamic nucleus; UPDRS, Unified Parkinson's Disease Rating Scale.
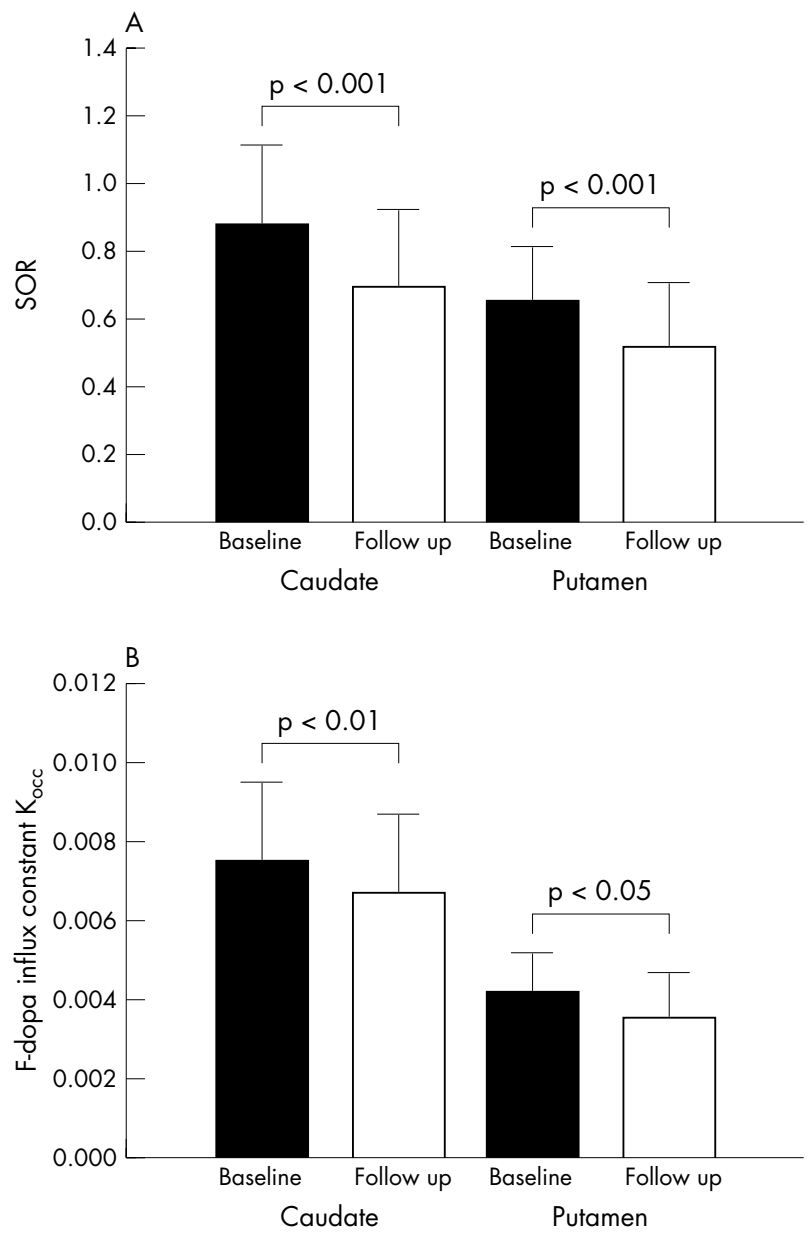

Figure 1 Significant reduction of the striatal F-dopa uptake from baseline to follow up PET calculated with (A) the striatal to occipital ratio $(S O R ; n=30)$ and $(B)$ the F-dopa influx constant $\left(K_{o c c} ; n=20\right)$ methods. The $\mathrm{p}$ values are for mean comparisons with paired $t$ test statistics. the caudate nucleus (table 2 ). The mean striatal $\mathrm{K}_{\mathrm{occ}}$ and SOR values decreased significantly during the follow up period (table 2 and fig 1). The annual decline of $\mathrm{K}_{\mathrm{occ}}$ was $0.0007 \mathrm{l}$ (0.00081) $\mathrm{min}^{-1}$ in the caudate and $0.00049(0.00074) \mathrm{min}^{-1}$ in the putamen (table 3). Likewise, SOR values decreased by 0.11 (0.10) per year in the caudate and by $0.08(0.09)$ per year in the putamen. These rates of decline were reflected in the annual progression rates relative to baseline given in table 3 . Progression data based on SOR values were nearly identical in the two study centres (table 3). No significant correlation was found between PET progression rates determined by either method and the clinical deterioration measured with UPDRS motor scale (data not shown).

\section{DISCUSSION}

This prospective, two centre F-dopa PET study is the first to measure disease progression objectively in PD patients with clinically effective bilateral STN DBS. In a total of 30 patients we found a significant decrease of striatal F-dopa uptake over the 16 month follow up period which corresponds to progression rates ranging from $9.5 \%$ to $12.9 \%$ loss of baseline radiotracer binding per year depending on the approach used for PET data analysis. Therefore, our data are the first to prove a continuing decline in dopaminergic function even in PD subjects with effective STN stimulation.

Our data are in good agreement with disease progression rates reported in previous studies on medically treated PD patients establishing F-dopa PET as a valid tool for measuring the progression of $\mathrm{PD}^{28-30}$ For example, Morrish and colleagues found an average annual decline of $12.5 \%$ from baseline in the putamen and $4 \%$ from baseline in the caudate. $^{29}$ More recent single photon emission computed tomography (SPECT) and PET studies using the dopamine transporter ligands $\left[{ }^{123} \mathrm{I}\right] \beta$-CIT and [F]CFT have also reported a $4-12.5 \%$ progression from baseline in the caudate nucleus and $8-13.1 \%$ in the putamen. ${ }^{14}{ }^{31}$ However, most of these studies investigated PD patients in earlier stages of the disease compared with our STN stimulated cohort. Therefore, our results are the first to show a comparable rate of dopaminergic deterioration in PD patients with a long disease duration and severe motor complications at the time of baseline PET. In line with the studies cited above, we found high interindividual variability in the rates of disease 
Table 2 Striatal F-dopa influx constants $\left(\mathrm{K}_{\text {occ }}\right)$ and SOR values at baseline and follow up PET scans $(n=30$; values are mean (SD))

\begin{tabular}{|c|c|c|c|c|}
\hline & \multicolumn{2}{|c|}{ Baseline F-dopa PET } & \multicolumn{2}{|c|}{ Follow up F-dopa PET } \\
\hline & $K_{\text {occ }}\left(\min ^{-1}\right)$ & SOR & $K_{\text {occ }}\left(\min ^{-1}\right)$ & SOR \\
\hline \multicolumn{5}{|l|}{ Caudate } \\
\hline Cologne & $0.0076(0.0021)$ & $0.85(0.20)$ & $0.0067(0.0020)$ & $0.71(0.21) \dagger$ \\
\hline Groningen & - & $0.94(0.32)$ & - & $0.65(0.27) \ddagger$ \\
\hline Total & $0.0076(0.0021)$ & $0.88(0.24)$ & $0.0067(0.0020)^{* *}$ & $0.69(0.23)^{* * *}$ \\
\hline \multicolumn{5}{|l|}{ Putamen } \\
\hline Cologne & $0.0042(0.0010)$ & $0.65(0.15)$ & $0.0036(0.0011)$ & $0.55(0.19) \dagger$ \\
\hline Groningen & - & $0.68(0.19)$ & - & $0.49(0.20) \ddagger$ \\
\hline Total & $0.0042(0.0010)$ & $0.66(0.16)$ & $0.0036(0.0011)^{*}$ & $0.52(0.19)^{\star \star \star}$ \\
\hline \multicolumn{5}{|c|}{$\begin{array}{l}\text { Difference between total baseline and follow up values: }{ }^{*} p<0.05,{ }^{* *} p<0.01,{ }^{* *} p<0.001 \text { (paired } t \text { test). } \\
\text { Length of the follow up period (mean (SD)): }+15.6(3.6) \text { months }(n=20), \neq 27.0(8.8) \text { months }(n=10) \text {. } \\
\text { Normal ranges in healthy controls: SOR caudate } 1.68(0.25), S O R \text { putamen } 1.69(0.29)(n=7, \text { age } 56 \text { (9) years); } \\
\mathrm{K}_{\text {occ }} \text { caudate } 0.0125(0.0015) \text { min }^{-1}, \mathrm{~K}_{\text {occ }} \text { putamen } 0.0126(0.0013) \text { min }^{-1}(\mathrm{n}=16 \text {, age } 54 \text { (12) years). } \\
\mathrm{K}_{\text {occ }} \text { F-dopa influx constant; PET, positron emission tomography; SOR, striatal to occipital ratio. }\end{array}$} \\
\hline
\end{tabular}

progression in our STN stimulated patients, reflected in the high standard deviations and 95\% confidence intervals. Differences in PET data acquisition and analysis can be mostly disregarded as causal factors in view of the nearly identical results in both study centres. As the two PET scans for each patient were taken in an identical manner with the same PET camera at both centres, methodological differences should not have influenced the percentage baseline progression values. Thus, our data corroborate the high interindividual biological variability in the speed of dopaminergic degeneration even in advanced PD patients.

Our PET data are also in line with two recent long term follow up studies on STN DBS which reported a slight increase in UPDRS motor scores over two and five years, in particular a worsening of axial symptoms subscores such as akinesia, postural instability, and freezing of gait. $^{56}$ In contrast, Herzog et al found no pertinent deterioration of UPDRS scores in their two year follow up of 20 STN DBS patients. ${ }^{4}$ However, it has to be kept in mind that clinical data on PD progression are often influenced by hangover effects of medication on the "drug-off, stimulation-on" motor performance and also by DBS-on the "drug-off, stimulation-off" condition. ${ }^{32}$ Therefore the most valuable aspect of our study is the objective monitoring of dopaminergic decline with Fdopa as a PET biomarker. We also found a slight and, with respect to the drug-on data, significant deterioration of the UPDRS scores over time. The absence of correlation of clinical and PET-based progression rates is in line with previous longitudinal F-dopa PET studies in medically treated PD patients. ${ }^{33}$ Perhaps the main reason for this is the effective long term control of the signs of dopamine deficiency by STN stimulation in the "drug-off, stimulation-on" condition, but our PET data suggest that DBS masks rather than prevents clinical PD progression.
We are aware that our study does not directly disprove a neuroprotective effect of STN DBS due to the small sample size and a lack of randomised design (progression determination in the DBS and a medically treated control group). However, we did not consider this study design for ethical reasons because the empirically well proved symptomatic relief of PD symptoms by STN DBS would have had to be withheld in severely handicapped patients for at least one year. Thus, our data merely verify an ongoing significant loss of dopaminergic function within the first two years of STN stimulation in advanced PD which, however, makes a clinically relevant neuroprotective effect of STN stimulation highly unlikely.

The assumption about neuroprotective properties of STN stimulation was essentially based on its putative blocking of glutamatergic STN hyperactivity, which is considered as an important aetiological factor for excitotoxicity and cell death within the substantia nigra. ${ }^{8}$ Although the reasons for the lack of a protective effect of STN DBS remain unclear, our findings can be interpreted as indirect arguments against the neurone inhibiting theory of DBS. Recent PET studies ${ }^{20}{ }^{34}$ that showed a local increase of energy metabolism in the midbrain and the STN target area under effective STN DBS suggesting activation rather than blocking stimulation support this view. Recent animal studies have demonstrated increased glutamate levels in the internal pallidum of normal rats and elevated firing rates of internal pallidum neurones in parkinsonian monkeys during chronic STN stimulation. ${ }^{35} 36$ These data on vascular and metabolic activation in the DBS target region and its projection sites suggest that STN stimulation might result in a high-frequent, tonic, and regular neuronal activity pattern (so-called neuronal jamming) that replaces an abnormally synchronised and oscillating basal ganglion firing, characteristic of the parkinsonian state. ${ }^{3738}$ Thus, it is probable that the glutamatergic transmission as well

Table 3 Annual reduction of striatal $\mathrm{K}_{\mathrm{occ}}$ and $\mathrm{SOR}$ values and disease progression rates from baseline $(n=30$; values are mean (SD))

\begin{tabular}{|c|c|c|c|c|c|c|}
\hline & \multicolumn{2}{|l|}{ Annual reduction } & \multicolumn{4}{|c|}{ Annual disease progression from baseline (\%) } \\
\hline & $\mathrm{K}_{\text {occ }}\left(\min ^{-1}\right)$ & SOR & $\mathrm{K}_{\mathrm{occ}}$ & $95 \% \mathrm{Cl}$ & SOR & $95 \% \mathrm{Cl}$ \\
\hline \multicolumn{7}{|l|}{ Caudate } \\
\hline Cologne & $0.00071(0.00081)$ & $0.10(0.10)$ & $9.5(12.4)$ & $3.2-14.4$ & $12.2(12.6)$ & $6.4-18.1$ \\
\hline Groningen & - & $0.11(0.10)$ & - & - & $11.8(10.0)$ & $4.6-18.9$ \\
\hline Total & $0.00071(0.00081)$ & $0.11(0.10)$ & $9.5(12.4)$ & 3.2.- -14.4 & $12.1(11.6)$ & $7.7-16.4$ \\
\hline \multicolumn{7}{|l|}{ Putamen } \\
\hline Cologne & $0.00049(0.00074)$ & $0.08(0.09)$ & $10.7(17.9)$ & $2.5-18.3$ & $12.8(15.5)$ & $5.5-20.0$ \\
\hline Groningen & - & $0.08(0.08)$ & - & - & $11.8(12.4)$ & $2.9-20.6$ \\
\hline Total & $0.00049(0.00074)$ & $0.08(0.09)$ & $10.7(17.9)$ & $2.5-18.3$ & $12.4(14.3)$ & $7.1-17.8$ \\
\hline
\end{tabular}


as the excitotoxic drive from STN to the substantia nigra is not diminished by chronic STN stimulation alone.

From a clinical point of view, our PET data may help in maintaining realistic expectations about STN stimulationthat is, there is strong symptomatic relief but not a slowing of disease progression or even a cure of the disorder. The data also highlight the need for careful presurgical assessment of potential DBS candidates with respect to realistic treatment goals and the unchanged progressive character of PD after surgery. However, it has to be kept in mind that our study failed to show mitigation of disease progression in advanced PD patients with STN stimulation only. Our results cannot rule out that STN DBS might have a disease modifying effect in the earlier stages of $\mathrm{PD}$, which might help prevent the development of severe on-off fluctuations and levodopa induced dyskinesias in these patients. Future research is needed on this important question.

In conclusion, this is the first PET study to demonstrate that decline of dopaminergic function in advanced PD continues despite clinically effective bilateral STN stimulation. Therefore, neuroprotective properties of DBS in the STN target could not be confirmed. Nevertheless, it has to be emphasised that STN DBS is a very effective treatment option that offers favourable long term symptomatic relief to advanced PD patients with treatment fluctuations or an otherwise intractable tremor. Further research is needed to investigate whether DBS of subcortical targets may still have a role to play in the more comprehensive neuroprotective treatment for PD.

\section{ACKNOWLEDGEMENTS}

We thank all the staff of the Cologne and Groningen PET centres for their excellent technical assistance.

\section{Authors' affiliations}

R Hilker, L Burghaus, K Herholz, W-D Heiss, Department of Neurology, Medical University of Cologne, Cologne, Germany

A T Portman, T van Laar, R P Maguire, B M de Jong, K L Leenders, Department of Neurology, University Hospital, Groningen, the Netherlands

J Voges, A Koulousakis, V Sturm, Department of Stereotaxy and Functional Neurosurgery, Medical University of Cologne, Cologne, Germany

M J Staal, Department of Neurosurgery, University Hospital, Groningen, the Netherlands

J Pruim, PET Centre, University Hospital, Groningen, the Netherlands W-D Heiss, Max-Planck-Institute for Neurological Research Cologne, Cologne, Germany

This study was supported by the Stichting Internationaal Parkinson Fonds, Hoofddorp (the Netherlands).

Competing interests: none declared

The study was approved by local ethics committees of Cologne and Groningen medical faculties.

\section{REFERENCES}

1 Limousin $P$, Krack $P$, Pollak $P$, et al. Electrical stimulation of the subthalamic nucleus in advanced Parkinson's disease. N Engl J Med 1998;339:1 105-11.

2 Benabid AL, Koudsie A, Benazzouz A, et al. Deep brain stimulation for Parkinson's disease. Adv Neurol 2001;86:405-12.

3 Ashkan K, Wallace B, Bell BA, Benabid AL. Deep brain stimulation of the subthalamic nucleus in Parkinson's disease 1993-2003: where are we 10 years on? Br J Neurosurg 2004;18:19-34.

4 Herzog J, Volkmann J, Krack P, et al. Two-year follow-up of subthalamic deep brain stimulation in Parkinson's disease. Mov Disord 2003;18:1332-7.

5 Krack P, Batir A, Van Blercom N, et al. Five-year follow-up of bilateral stimulation of the subthalamic nucleus in advanced Parkinson's disease. N Engl J Med 2003;349:1925-34.

6 Kleiner-Fisman G, Fisman DN, Sime E, et al. Long-term follow up of bilateral deep brain stimulation of the subthalamic nucleus in patients with advanced Parkinson disease. J Neurosurg 2003;99:489-95.

7 Rodriguez-Oroz MC, Gorospe A, Guridi J, et al. Bilateral deep brain stimulation of the subthalamic nucleus in Parkinson's disease. Neurology 2000;55:S45-S51.
8 Rodriguez MC, Obeso JA, Olanow CW. Subthalamic nucleus-mediated excitotoxicity in Parkinson's disease: a target for neuroprotection. Ann Neurol 1998;44:S175-S88

9 Obeso JA, Rodriguez-Oroz MC, Rodriguez M, et al. Pathophysiologic basis of surgery for Parkinson's disease. Neurology 2000;55:S7-S12.

10 Benazzouz A, Piallat B, Ni ZG, et al. Implication of the subthalamic nucleus in the pathophysiology and pathogenesis of Parkinson's disease. Cell Transplant 2000;9:215-21.

11 Piallat B, Benazzouz A, Benabid AL. Subthalamic nucleus lesion in rats prevents dopaminergic nigral neuron degeneration after striatal 6-OHDA injection: behavioural and immunohistochemical studies. Eur J Neurosci 1996;8:1408-14

12 Piallat B, Benazzouz A, Benabid AL. Neuroprotective effect of chronic inactivation of the subthalamic nucleus in a rat model of Parkinson's disease. J Neural Transm Suppl 1999;55:71-7.

13 Nakao N, Nakai E, Nakai K, et al. Ablation of the subthalamic nucleus supports the survival of nigral dopaminergic neurons after nigrostriatal lesions induced by the mitochondrial toxin 3-nitropropionic acid. Ann Neurol 1999;45:640-51.

14 Nurmi E, Ruottinen HM, Kaasinen V, et al. Progression in Parkinson's disease: a positron emission tomography study with a dopamine transporter ligand [18F]CFT. Ann Neurol 2000;47:804-8.

15 Morrish PK, Sawle GV, Brooks DJ. The rate of progression of Parkinson's disease. A longitudinal [18F]DOPA PET study. Adv Neurol 1996;69:427-31.

16 Leenders KL, Palmer AJ, Quinn N, et al. Brain dopamine metabolism in patients with Parkinson's disease measured with positron emission tomography. J Neurol Neurosurg Psychiatry 1986;49:853-60.

17 Hoehn MM, Yahr MD. Parkinsonism: onset, progression and mortality. Neurology 1967; 17:427-42.

18 Hughes AJ, Daniel SE, Kilford L, et al. Accuracy of clinical diagnosis of idiopathic Parkinson's disease: a clinico-pathological study of 100 cases. J Neurol Neurosurg Psychiatry 1992;55:181-4.

19 Defer GL, Widner H, Marie RM, et al. Core assessment program for surgical interventional therapies in Parkinson's disease (CAPSIT-PD). Mov Disord 1999; 14:572-84.

20 Hilker R, Voges J, Weisenbach S, et al. Subthalamic nucleus stimulation restores glucose metabolism in associative and limbic cortices and in cerebellum: evidence from a FDG-PET study in advanced Parkinson's disease. J Cereb Blood Flow Metab 2004;24:7-16.

21 Voges J, Volkmann J, Allert N, et al. Bilateral high-frequency stimulation in the subthalamic nucleus for the treatment of Parkinson disease: correlation of therapeutic effect with anatomical electrode position. J Neurosurg 2002;96:269-79.

22 Fahn S, Elton RL. Unified Parkinson's Disease Rating Scale. In: Fahn S, Marsden CD, Calne D, Goldstein M, eds. Recent development in Parkinson's disease. Florham Park, NJ: Mac-Millan Health Care Information, 1987:153-63.

23 Wienhard K, Dahlbom M, Eriksson L, et al. The ECAT EXACT HR: performance of a new high resolution positron scanner. J Comput Assist Tomogr 1994; 18:110-18.

24 Wienhard K, Eriksson L, Grootoonk S, et al. Performance evaluation of the positron scanner ECAT EXACT. J Comput Assist Tomogr 1992;16:804-13.

25 Pietrzyk U, Herholz K, Fink G, et al. An interactive technique for threedimensional image registration: validation for PET, SPECT, MRI and CT brain studies. J Nucl Med 1994;35:2011-18.

26 Dhawan V, Ma Y, Pillai V, et al. Comparative analysis of striatal FDOPA uptake in Parkinson's disease: ratio method versus graphical approach. J Nucl Med 2002;43:1324-30.

27 Patlak CS, Blasberg RG. Graphical evaluation of blood-to-brain transfer constants from multiple-time uptake data. Generalizations. J Cereb Blood Flow Metab 1985:5:584-90.

28 Morrish PK, Rakshi JS, Bailey DL, et al. Measuring the rate of progression and estimating the preclinical period of Parkinson's disease with [18F]dopa PET. J Neurol Neurosurg Psychiatry 1998;64:314-19.

29 Morrish PK, Sawle GV, Brooks DJ. An [18F]dopa-PET and clinical study of the rate of progression in Parkinson's disease. Brain 1996;1 19:585-91.

30 Vingerhoets FJ, Snow BJ, Lee CS, et al. Longitudinal fluorodopa positron emission tomographic studies of the evolution of idiopathic parkinsonism. Ann Neurol 1994;36:759-64.

31 Marek K, Innis R, van Dyck C, et al. [123I]beta-CIT SPECT imaging assessment of the rate of Parkinson's disease progression. Neurology 2001;57:2089-94.

32 Nutt JG, Carter JH, Lea ES, et al. Evolution of the response to levodopa during the first 4 years of therapy. Ann Neurol 2002;51:686-93.

33 Brooks DJ. Monitoring neuroprotection and restorative therapies in Parkinson's disease with PET. J Neural Transm Suppl 2000:125-37.

34 Hilker R, Voges J, Burghaus L, et al. Deep brain stimulation of the STN activates the electrode target area in patients with advanced Parkinson's disease. Mov Disord 2004; 19:S300.

35 Windels F, Bruet N, Poupard A, et al. Effects of high frequency stimulation of subthalamic nucleus on extracellular glutamate and GABA in substantia nigra and globus pallidus in the normal rat. Eur J Neurosci 2000;12:4141-6.

36 Hashimoto T, Elder CM, DeLong MR, et al. Responses of pallidal neurons to electrical stimulation of the subthalamic nucleus in experimental primates. Mov Disord 2001;15:S31.

37 Vitek JL. Mechanisms of deep brain stimulation: excitation or inhibition. Mov Disord 2002;17:S69-72.

38 Miller WC, DeLong MR. Parkinsonian symptomatology. An anatomical and physiological analysis. Ann N Y Acad Sci 1988;515:287-302. 